\title{
Newest data on fluoroquinolone resistance mechanism of Shigella flexneri isolates in Jiangsu Province of China
}

Tingting Qin ${ }^{1 \dagger}$, Huimin Qiann ${ }^{2+}$, Wenting Fan ${ }^{3}$, Ping Ma ${ }^{1,3}$, Lu Zhou ${ }^{2}$, Chen Dong ${ }^{2}$, Bing Gu ${ }^{1,3^{*}}$ and Xiang Huo ${ }^{2^{*}}$

\begin{abstract}
Background: To determine the prevalence, antimicrobial susceptibility patterns and related presence of mutations in quinolone resistance-determining region (QRDR) genes and plasmid-mediated quinolone resistance (PMQR) among Shigella flexneri isolates obtained from Jiangsu Province, China.

Methods: A total of 400 Shigella flexneri clinical isolates collected during 2012-2015 were identified by biochemical and serological methods, and the antimicrobial susceptibility pattern was evaluated using the disc-diffusion method. PCR and DNA sequencing were accomplished to identify mutations in gyrA, gyrB, parC and parE, and the

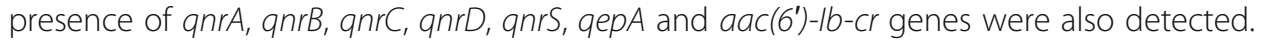

Results: Of all the Shigella flexneri, $75.8 \%$ were resistant to nalidixic acid, and $37.0 \%$ were categorized as norfloxacin resistant. Overall, 75.5\% of isolates possessed gyrA mutations (Ser83Leu, Asp87Gly/Asn and His211Tyr), while 84.3\% had parC mutations (Ser80lle, Ala81Pho, Gln91His and Ser129Pro). The most prevalent point mutations in gyrA and parC were Ser83Leu (75.5\%, 302/400) and Ser80lle (74.5\%, 298/400), relatively. Besides, the Gln517Arg alternation in gyrB was detected in $13 \mathrm{~S}$. flexneri isolates and no mutations were identified in parE. PMQR determinations of anrB, gnrS and $a a c\left(6^{\prime}\right)-1 b-c r$ were detected among 16 strains (4.0\%).

Conclusions: The results presented here show that fluoroquinolone resistance in these clinical isolates result from mutations in chromosome, besides, despite the low prevalence of PMQR determinants in Jiangsu, it is essential to continue surveillance PMQR determinants in this area.
\end{abstract}

Keywords: Shigella flexneri, gyrA, Plasmid, anrS, Fluoroquinolone resistance

\section{Background}

Shigella, a common cause of diarrheal disease worldwide, is a significant public health burden, particularly in sub-Saharan Africa and Asia [1]. In China, morbidity due to diarrhoea has decreased from 84 million in 1988 episodes to approximately $0.8-1.7$ million in 2000 [2], despite that, diarrhoeal diseases remain sixth in the rank of death caused by infectious disease in China. Moreover, Walker CL et al. reported that China is among the 15 high-burden countries where nearly three-quarters of

\footnotetext{
* Correspondence: gb20031129@163.com; huox@foxmail.com

${ }^{\dagger}$ Equal contributors

${ }^{1}$ Department of Laboratory Medicine, Affiliated Hospital of Xuzhou Medical University, Xuzhou 221002, China

${ }^{2}$ Jiangsu Provincial Center for Disease Control and Prevention, Nanjing

210009, China

Full list of author information is available at the end of the article
}

diarrhoea mortality concentrated in [3]. Symptoms caused by Shigella infection ranges from fever and fatigue to diarrhoea and/or dysentery, and frequent mucoid bloody stools, abdominal cramps and tenesmus occurred within hours to days [4].

Among the four species of Shigella (Shigella flexneri, Shigella sonnei, Shigella boydii and Shigella dysenteriae), S. flexneri is the predominant species in developing countries and the most frequent cause of bacterial dysentery [5]. Presently, fluoroquinolones emerged as the preferred agents for treatment of Shigella infection, however, the progressive increase in antimicrobial resistance further narrows the choice of effective antimicrobials. Around China, an increase in the occurrence of antimicrobial resistance have been reported, resistance rate to norfloxacin was $22.3 \%$ in a surveillance between 2005 and 
2011 in Anhui Province [6]. In our previous study, the proportion of norfloxacin-resistant S. flexneri was as high as $36.8 \%$ in the period 2006-2011 in Jiangsu Province [7].

The development of fluoroquinolone resistant was found to be associated with several mechanisms that have been documented, primarily attributed to multiple mutations in the quinolone resistance-determining region (QRDR) of the genes encoding DNA gyrase (gyrA and $g y r B$ ) and topoisomerase IV (parC and parE) [8], additionally, plasmid-mediated quinolone resistance (PMQR) and efflux pump mediators mechanisms have been identified related to resistance [9].

In our previous survey of $S$. flexneri isolated from 2001 to 2011, fluoroquinolone resistance profiles and the presence of QRDR genes encoding resistance to fluoroquinolone have been described, in this study, we wanted to assess the level of antimicrobial resistance and fluoroquinolone resistance mechanisms including QRDR and PMQR genes of $S$. flexneri collected from patients between 2012 and 2015 in Jiangsu Province, China. Indeed, this is the first work to detect PMQR determinants in Shigella flexneri strains from Jiangsu Province.

\section{Methods}

\section{Strains collection and examination}

A total of 400 non-duplicate, consecutive clinical $S$. flexneri strains were collected from Jiangsu Provincial Center for Disease Control and Prevention between January 2012 and December 2015, and the isolates enrolled were isolated throughout Jiangsu Province. Jiangsu Province is a region with approximately 100 thousand square kilometers and a population of 75 million and 13 cities located in this area. The cities were Nanjing $(n=9)$, Suzhou $(n=44)$, Wuxi $(n=22)$, Zhenjiang $(n=20)$, Changzhou $(n=40)$, Nantong $(n=27)$, Taizhou $(n=60)$, Yangzhou $(n=18)$, Xuzhou $(n=33)$, Yancheng $(n=25)$, Lianyungang $(n=44)$, Huai'an $(n=26)$, and Suqian $(n=32)$.

Samples were cultured for Shigella by streaking diarrheal stools directly onto Salmonella-Shigella agar and then incubated at $37{ }^{\circ} \mathrm{C}$ for 16 to $24 \mathrm{~h}$. After culture and screening, the isolates were identified using VITEK 2 system (bioMérieux, Marcy l’Etoile, France).

\section{Serotyping}

Serologic identification was performed by slide agglutination with polyvalent somatic $(\mathrm{O})$ antigen grouping sera, followed by testing with available monovalent antisera for specific identification of serotypes according to the manufacturer's instructions (Denka Seiken, Japan).

\section{Drug susceptibility test}

Antibiotic susceptibility of the Shigella isolates was tested using the Kirby-Bauer method on Muller-Hinton agar plates for nalidixic acid (NAL) and norfloxacin (NOR) (Oxoid, Hampshire, UK), results were interpreted according to Clinical and Laboratory Standards Institute (CLSI) performance standards [10]. A control strain of Escherichia coli (ATCC 25922) used for antibiotic susceptibility testing quality control.

Detection of point mutations in gyrA, gyrB, parC, and parE Preparation of bacterial DNA templates and PCR amplification of the quinolone resistance-determining regions of $\operatorname{gyr} A, \operatorname{gyr} B, \operatorname{parC}$ and $\operatorname{parE}$ were performed using primers and conditions as previously described (Table 1 ) [11-13]. The presence of the target genes was confirmed through $1.5 \%$ agarose gel electrophoresis. Subsequently, the amplification products of QRDR genes were purified, then, DNA sequencing was performed using primers (Genewiz, Suzhou, China). The sequence data were analyzed by comparison with sequences in GenBank.

\section{Detection of PMQR}

Besides, all isolates were screened for plasmid-mediated quinolone resistance (PMQR) genes ( $q n r A$, qnrB, qnrC, $q n r D, q n r S$, qepA and $\left.a a c\left(6^{\prime}\right)-I b-c r\right)$ as described in previous reports (Table 1) [14-18]. Next, the amplified PCR products were identified by agarose gel electrophoresis and the positive products were purified and sequenced (Genewiz, Suzhou, China) to confirm the presence of PMQR genes.

\section{Statistical analysis}

For analysis of resistance patterns and mutation characteristics, statistical comparisons were performed using the chi-Square test. The differences among the groups were considered to be statistically significant at $P<0.05$.

\section{Results}

\section{Bacterial isolation}

A number of $400 \mathrm{~S}$. flexneri isolates were isolated and identified from patients over the period from 2012 to 2015 in Jiangsu Province of China. The number of isolated strains of the four years accounted for 120, 114, 85 and 81, relatively, showing a decreasing trend. Among these clinical S. flexneri isolates, 2a $(n=156$, $39.0 \%)$ was the most prevalent serotype, followed by 1a $(n=75,18.8 \%), 2 \mathrm{~b}(n=66,16.5 \%), 1 \mathrm{~b}(n=61,15.3 \%)$ and $\mathrm{X}(n=18,39.0 \%)$. Other serotypes including $3 \mathrm{~b}, 4$, $4 \mathrm{a}, 4 \mathrm{c}$ and $\mathrm{Y}$ were also detected (Fig. 1).

\section{Fluoroquinolone susceptibility testing}

Of the $400 \mathrm{~S}$. flexneri isolates, $75.8 \%$ (303) were resistant to nalidixic acid, and $37.0 \%$ (148) were categorized as norfloxacin resistant. During the four years, resistance rate of nalidixic acid fluctuated between $70.0 \%$ and $80.0 \%$ and no clear changing trend was observed. A 
Table 1 Primes used in this study

\begin{tabular}{|c|c|c|c|}
\hline Gene & Sequence $\left(5^{\prime}-3^{\prime}\right)$ & Product size (bp) & Annealing temperature $\left({ }^{\circ} \mathrm{C}\right.$ \\
\hline gyrA & $\begin{array}{l}\text { F: TACACCGGTCAACATTGAGG } \\
\text { R: TTAATGATTGCCGCCGTCGG }\end{array}$ & 648 & 60 \\
\hline gyrB & $\begin{array}{l}\text { F: CAAACTGGCGGACTGTCAGG } \\
\text { R: TTCCGGCATCTGACGATAGA }\end{array}$ & 345 & 60 \\
\hline parc & $\begin{array}{l}\text { F: GTCTGAACTGGGCCTGAATGC } \\
\text { R: AGCAGCTCGGAATATTTCGACAA }\end{array}$ & 248 & 60 \\
\hline parE & $\begin{array}{l}\text { F: ATGCGTGCGGCTAAAAAAGTG } \\
\text { R: TCGTCGCTGTCAGGATCGATAC }\end{array}$ & 289 & 60 \\
\hline qnrA & $\begin{array}{l}\text { F: ATTTCTCACGCCAGGATTTG } \\
\text { R: GATCGGCAAAGGTTAGGTCA }\end{array}$ & 515 & 50 \\
\hline anrB & $\begin{array}{l}\text { F: GATCGTGAAAGCCAGAAAGG } \\
\text { R: ACGATGCCTGGTAGTTGTCC }\end{array}$ & 468 & 54 \\
\hline anrc & $\begin{array}{l}\text { F: GGGTTGTACATTTATTGAATC } \\
\text { R: TCCACTITACGAGGTTCT }\end{array}$ & 446 & 50 \\
\hline qnrD & $\begin{array}{l}\text { F: CGAGATCAATTTACGGGGAATA } \\
\text { R: AACAAGCTGAAGCGCCTG }\end{array}$ & 581 & 50 \\
\hline anrs & $\begin{array}{l}\text { F: ACGACATTCGTCAACTGCAA } \\
\text { R: TAAATTGGCACCCTGTAGGC }\end{array}$ & 416 & 55 \\
\hline$a a c\left(6^{\prime}\right)-1 b-c r$ & $\begin{array}{l}\text { F: GCAACGCAAAAACAAAGTTAGG } \\
\text { R: GTGTTTGAACCATGTACA }\end{array}$ & 560 & 55 \\
\hline qepA & $\begin{array}{l}\text { F: GCAGGTCCAGCAGCGGGTAG } \\
\text { R: CTTCCTGCCCGAGTATCGTG }\end{array}$ & 217 & 60 \\
\hline
\end{tabular}

comparative analysis of the resistance against norfloxacin performed illustrated that the proportion of norfloxacin resistant isolates between $2014(36 / 85)$ and 2015 (33/81) was notably higher than that isolated in 2012 $(41 / 120)$ and $2013(38 / 114)(P<0.05)$. Fluoroquinolone susceptibility profiles of the isolates also varied between serotypes. Serotype 1a showed the highest resistance rate to both nalidixic acid and norfloxacin, with resistance rates of $88.0 \%$ and $46.7 \%$, respectively, and the proportions were closely followed by serotype $2 \mathrm{a}$ of $85.9 \%$ and 45.5\%. Most noticeably was that all the S. flexneri isolates that identified as serotype $4,4 \mathrm{a}, 4 \mathrm{c}, \mathrm{X}$ and $\mathrm{Y}$ were nalidixic acid resistant and almost half of the serotype $4 \mathrm{c}$ isolates were resistant to norfloxacin.

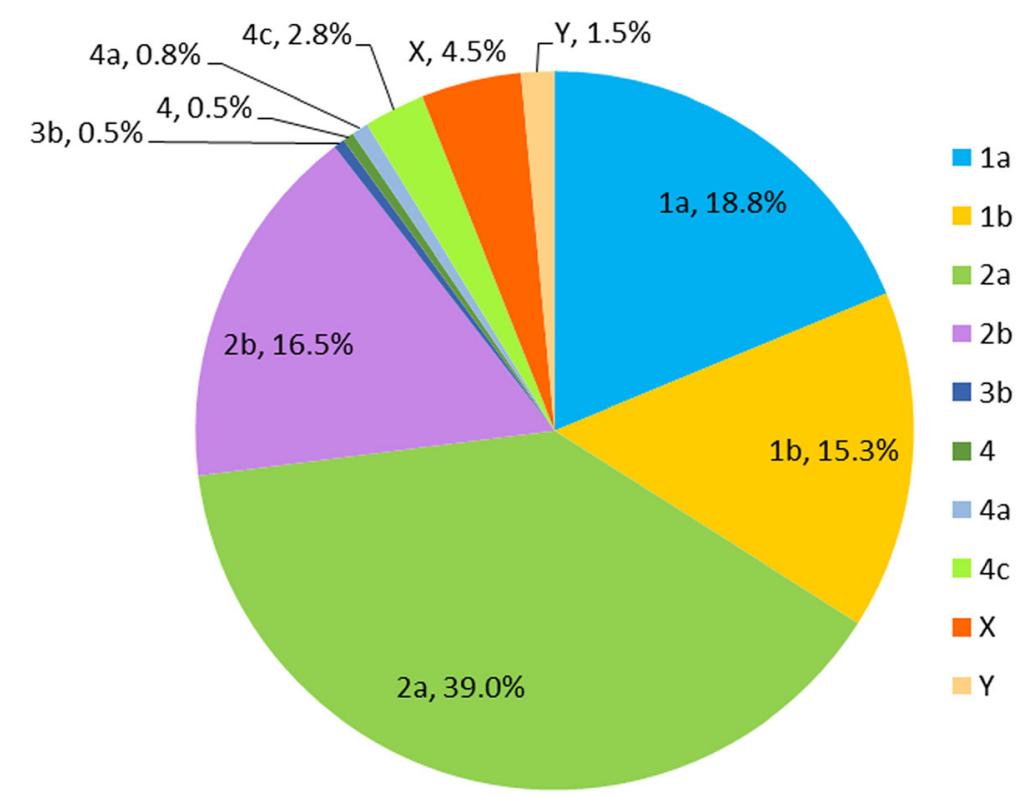

Fig. 1 Serotypes of the Shigella flexneri strains 


\section{Mutations in DNA gyrase and topoisomerase IV genes Mutations in gyrA gene}

DNA sequencing results showed that $75.5 \%$ (302/400) of isolates possessed gyrA mutations, and all these isolates were detected with Ser83Leu. Most of the S. flexneri isolates (295/302) were detected with two or more mutations in gyrA, 229 isolates displayed Ser83Leu, Asp87Gly/Asn and His211Tyr simultaneously, 66 isolates displayed both Ser83Leu and His211Tyr. Additionally, the mutation of Ser83Leu and His211Tyr took up a stable share during the four years while the association between time distribution and amino acid substitution at codon 87 in the parC gene was statistically significant $(P<0.05)$ (Table 2$)$. A significant annually decrease in the substitution of Asp-87 to Gly was seen $(40.8 \%$ in 2012 to $13.6 \%$ in 2015 ) whereas the mutation rate of Asp-87 to Asn remained stable in the first two years and fluctuated largely in 2014 (Table2).

\section{Mutations in parC gene}

For the parC gene, 84.3\% (337/400) had mutations, 298 and 152 isolates carried substitution at codon Ser80 and Ser129, relatively. Of those strains, 113 showed double mutations of Ser80Ile and Ser129Pro, remarkably, these 113 isolates were also observed with mutations at codon Ser83 and His211 in gyrA. Addtionally, Gln91His substitution in $\operatorname{parC}$ was detected in 2 isolates from 2012 and one of the two also revealed the amino acid substitution Ala81Pho in parC. During the four years, the mutation rate of Ser80Ile ranged between $67.9 \%$ and $77.5 \%$. In contrast, the mutation rate of Ser129Pro kept rising from 2012 to 2014, and then decreased to $24.7 \%$ in 2015 (Table 2).

\section{Mutation in gyrB and parE genes}

Among the S. flexneri isolates, 3.3\% (13/400) were detected with amino acid substitution at Gln-517 to Arg in $\operatorname{gyr} B$ and no mutations were identified in parE (Table 2). Furthermore, 10 out of the 13 alternation in gyrB gene showed another four point mutations in either gyrA or parC.

\section{Mutations and fluoroquinolone susceptibility}

There were $94.4 \%(286 / 303)$ of the nalidixic acid resistant S. flexneri carried Ser83Leu mutation (Table 3). Attentionally, obvious difference of Asp87Gly/Asn was observed between norfloxacin susceptible and resistant isolates $(P<0.05)$, Asp87Gly/Asn occurred more often among norfloxacin resistant S. flexneri. Another mutation in gyrA, the substitution His-211 to Tyr among fluoroquinolone susceptible was much lower than that in fluoroquinolone resistant strains. The Ser129Pro in parC was identified as no difference in fluoroquinolone susceptible and resistant S. flexneri according to statistical analysis $(P>0.05)$. A total of $81.8 \%(121 / 148)$ of the norfloxacin resistant $S$. flexneri contained at least three amino acid substitutions of Ser83Leu and Asp87Gly/Asn in gyrA and Ser80Ile in parC. Surprisingly, $11.8 \%$ (11/93) of the fluoroquinolone susceptible isolates also carried the three common mutations in gyrA and parC.

\section{Mutations and serotypes}

Also, the significant difference between serotypes and Asp-87 mutation in parC was observed. There were almost no mutations in $S$. flexneri serotype 1 b (Table 4). Among the common serotypes, the proportion of substitution Asp-87 to Asn in serotype 2a was much more (64.1\%) higher than that in other serotypes $(P<0.05)$. In contrast, the change of Asp-87 to Gly was highly prevalent in serotype $2 \mathrm{~b}(59.1 \%)$ and $1 \mathrm{a}$ (52.0\%), however, presenting a decreasing trend during 2012-2015.

\section{Characterization of PMQR genes}

PMQR determinants were detected alone or in combination in $4.0 \%$ (16 of 400) of isolates, qnrB, qnrS and $a a c\left(6^{\prime}\right)-I b-c r$ were present in $0.3 \%$ (1/400), 2.0\% (8/400) and $2.0 \%(8 / 400)$ of those isolates, respectively (Table 5), while the $q n r A, q n r C, q n r D$ and $q e p A$ genes were not detected. A qnrS positive isolate was detected in combination with $a a c\left(6^{\prime}\right)-I b-c r$. Except for the $q n r B$ positive isolate without any mutation, 15 of the 16 PMQR determinants positive isolates had at least two mutations of Ser83Leu in gyrA and Ser80Ile in parC gene. Of all the

Table 2 Mutations in gyrA, gyrB and parC genes in Shigella flexneri strains in the present study

\begin{tabular}{|c|c|c|c|c|c|}
\hline Mutations & $2012(n=120)$ & $2013(n=114)$ & $2014(n=85)$ & $2015(n=81)$ & Total $(n=400)$ \\
\hline Ser83Leu & $79.2(95)$ & $75.4(86)$ & $75.3(64)$ & $70.4(57)$ & 75.5 (302) \\
\hline Asp87Asn/ Gly & $65.0(78)$ & $50.9(58)$ & $61.2(52)$ & $50.6(41)$ & 57.3 (229) \\
\hline Asp87Asn & $24.2(29)$ & $24.6(28)$ & $41.2(35)$ & $37.0(30)$ & $30.5(122)$ \\
\hline Asp87 Gly & $40.8(49)$ & $26.3(30)$ & $20.1(17)$ & $13.6(11)$ & 26.8 (107) \\
\hline His211Tyr & $75.0(90)$ & $74.6(85)$ & $75.3(64)$ & $69.1(56)$ & 73.8 (295) \\
\hline Gln517Arg & $5.8(7)$ & $3.5(4)$ & $2.5(2)$ & $0.0(0)$ & $3.3(13)$ \\
\hline Ser80lle & $77.5(93)$ & $75.4(86)$ & $75.3(64)$ & $67.9(55)$ & 74.5 (298) \\
\hline Ser129Pro & $33.3(40)$ & $41.2(47)$ & $52.9(45)$ & $24.7(20)$ & $38.0(152)$ \\
\hline
\end{tabular}


Table 3 Mutations and fluoroquinolone susceptibility of Shigella flexneri strains

\begin{tabular}{lllll}
\hline Point mutations & $\begin{array}{l}\text { NAL-S \& NOR-S strains } \\
(n=93)\end{array}$ & $\begin{array}{l}\text { NAL-R strains } \\
(n=303)\end{array}$ & $\begin{array}{l}\text { NAL-R \& NOR-S strains } \\
(n=84)\end{array}$ & $\begin{array}{l}\text { NOR-R strains } \\
(n=148)\end{array}$ \\
\hline No mutation & $46(49.5)$ & $10(3.3)$ & $5(6.0)$ & $5(3.4)$ \\
GyrA-Ser83Leu & $14(15.1)$ & $286(94.4)$ & $77(91.7)$ & $138(93.2)$ \\
GyrA-Asp87Asn/Gly & $11(11.8)$ & $217(71.6)$ & $28(33.3)$ & $122(82.4)$ \\
GyrA-His211Tyr & $13(13.9)$ & $280(92.4)$ & $74(88.1)$ & $135(91.2)$ \\
GyrB-Gln517Arg & $3(3.2)$ & $10(3.3)$ & $3(3.6)$ & $3(2.0)$ \\
ParC-Ser80lle & $15(16.1)$ & $281(92.7)$ & $75(89.3)$ & $135(91.2)$ \\
ParC-Ser129Pro & $35(37.6)$ & $116(38.3)$ & $37(44.0)$ & $48(32.4)$ \\
GyrA-Ser83Leu \& ParC-Ser80lle & $13(13.9)$ & $280(92.4)$ & $75(89.3)$ & $134(90.5)$ \\
GyrA-Ser83Leu \& ParC-Ser80lle \& GyrA- & $11(11.8)$ & $216(71.3)$ & $28(33.3)$ & $121(81.8)$
\end{tabular}

$N A L-S$ nalidixic acid susceptible, NAL-R nalidixic acid resistant, NOR-S norfloxacin susceptible, NOR- $R$ norfloxacin resistant. ${ }^{\text {a }}$ NAL-S \& NOR-S strains, besides these strains, there were 3 strains showed NAL-S \& NOR-I (intermediate resistant/susceptible) and 1 NAL-I \& NOR-S strain. ${ }^{b}$ NAL-R strains, among these strains, there were 71 NAL-R \& NOR-I strains, which were not analysed in the table. The isolates that showed intermediate susceptible/resistant to NAL/NOR were not listed in detail in this table

PMQR determinants' positive isolates, 87.5\% (14/16) were resistant to nalidixic acid and $56.3 \%$ (9/16) of them were resistant to norfloxacin.

\section{Discussion}

Our data documented an ongoing decreasing trend of $S$. flexneri from 2012 to 2015, which is reflective of a current shift in the epidemiologic distribution of this specie in China in recent years $[19,20]$. The same tendency of S. flexneri observed not only in China but also in some other countries like Bangladesh and Thailand $[21,22]$. However, due to the unbalanced socioeconomic conditions, the distribution of Shigella infection species varies within the same country, a previous study indicated that lower rates of $S$. flexneri cases were observed in East, North and Northeast China, as compared to those in Middle, South, Northwest and Southwest China [19]. A retrospective study we conducted before have reported that 16 serotypes was observed between 2001 and 2011 in Jiangsu Province, with serovars 2a, 2b and 1a being the most common serotypes [7], in this study, we found that the top three most common $S$. flexneri serotypes were consistent with that years ago. Overall, both serotypes $4 \mathrm{c}$ and $\mathrm{X}$ accounted for a proportion in the last 15 years [7]. All this illustrated that the predominant serotypes $2 \mathrm{a}, 2 \mathrm{~b}$ and $1 \mathrm{a}$ remained important serotype, and distribution of some other serotypes fluctuated over the years. However, what's worrisome is that the only vaccine currently in use against $S$. flexneri in China is a live serotype $2 \mathrm{a}$ vaccine [23], hence a broadspectrum Shigella vaccine that protect against all the $S$. flexneri serotypes/subserotypes is necessary [5].

At present, the occurrence and prevalence of fluoroquinolone-resistant $S$. flexneri poses a great challenge for the effective treatment of shigellosis. Fluoroquinolone-resistant Shigella has been explicitly listed as one of the top concerns in the current international focus on antimicrobial resistance [24]. In this

Table 4 The Asp-87 mutation in gyrA in the four predominate Shigella flexneri serotypes

\begin{tabular}{|c|c|c|c|c|c|c|}
\hline \multirow[t]{2}{*}{ Serotypes } & \multirow[t]{2}{*}{ Mutation } & \multicolumn{4}{|l|}{ Years } & \multirow[t]{2}{*}{ Total } \\
\hline & & $2012\left(\%^{a}\right)$ & $2013\left(\%^{b}\right)$ & $2014\left(\%^{\mathrm{C}}\right)$ & $2015\left(\%{ }^{d}\right)$ & \\
\hline \multirow[t]{2}{*}{$2 \mathrm{a}(n=156)$} & gyrA-Asp87Asn & $59.6(28 / 47)$ & $53.7(22 / 41)$ & $63.6(21 / 33)$ & $82.9(29 / 35)$ & $64.1(100 / 156)$ \\
\hline & gyrA-Asp87Gly & $23.4(11 / 47)$ & $9.8(4 / 41)$ & $15.2(5 / 33)$ & $14.3(5 / 35)$ & $16.0(25 / 156)$ \\
\hline \multirow[t]{2}{*}{$1 a(n=75)$} & gyrA-Asp87Asn & $0.0(0 / 25)$ & $10.7(3 / 28)$ & $53.8(7 / 13)$ & $0.0(0 / 9)$ & $13.3(10 / 75)$ \\
\hline & gyrA-Asp87Gly & $88.0(22 / 25)$ & $39.3(11 / 28)$ & $23.1(3 / 13)$ & $33.3(3 / 9)$ & $52.0(39 / 75)$ \\
\hline \multirow[t]{2}{*}{$2 \mathrm{~b}(n=66)$} & gyrA-Asp87Asn & $0.0(0 / 21)$ & $10.5(2 / 19)$ & $8.3(1 / 12)$ & $7.1(1 / 14)$ & $6.1(4 / 66)$ \\
\hline & gyrA-Asp87Gly & $76.2(16 / 21)$ & $68.4(13 / 19)$ & $58.3(7 / 12)$ & $21.4(3 / 14)$ & $59.1(39 / 66)$ \\
\hline \multirow[t]{2}{*}{$1 b(n=61)$} & gyrA-Asp87Asn & $0.0(0 / 20)$ & $0.0(0 / 10)$ & $0.0(0 / 12)$ & $0.0(0 / 19)$ & $0.0(0 / 61)$ \\
\hline & gyrA-Asp87Gly & $0.0(0 / 20)$ & $0.0(0 / 10)$ & $0.0(0 / 12)$ & $0.0(0 / 19)$ & $0.0(0 / 61)$ \\
\hline
\end{tabular}

$\mathrm{a}_{\%}=$ Mutation strains/number of different serotypes in 2012

$\mathrm{b}_{\%}=$ Mutation strains/number of different serotypes in 2013

$c_{\%}=$ Mutation strains/number of different serotypes in 2014

$\mathrm{d}_{\%}=$ Mutation strains/number of different serotypes in 2015 
Table 5 Characteristics of the PMQR determinants positive Shigella flexneri isolates

\begin{tabular}{|c|c|c|c|c|c|c|c|c|c|c|c|c|}
\hline \multirow[t]{2}{*}{$\begin{array}{l}\text { Isolate } \\
\text { number }\end{array}$} & \multirow[t]{2}{*}{ Serotype } & \multirow[t]{2}{*}{$\begin{array}{l}\text { Year of } \\
\text { isolation }\end{array}$} & \multirow[t]{2}{*}{ City } & \multicolumn{2}{|c|}{$\begin{array}{l}\text { Antibiotic } \\
\text { susceptibility }\end{array}$} & \multicolumn{6}{|c|}{ Target gene mutations } & \multirow[t]{2}{*}{$\begin{array}{l}\text { PMQR } \\
\text { determinants }\end{array}$} \\
\hline & & & & NAL & NOR & gyrA & & & gyrB & parC & & \\
\hline F12002 & $3 b$ & 2012 & Nantong & $R$ & $R$ & & & & & & & $q n r B$ \\
\hline F12019 & $2 b$ & 2012 & Yangzhou & $\mathrm{R}$ & 1 & Ser83Leu & Asp87Gly & His211Tyr & & Ser80lle & & $a a c\left(6^{\prime}\right)-1 b-c r$ \\
\hline F12037 & $2 a$ & 2012 & Zhenjiang & $S$ & I & Ser83Leu & & His211Tyr & & Ser80lle & & $a a c\left(6^{\prime}\right)-1 b-c r$ \\
\hline F12066 & $4 c$ & 2012 & Xuzhou & $\mathrm{R}$ & R & Ser83Leu & & His211Tyr & & Ser80lle & & $a a c\left(6^{\prime}\right)-1 b-c r$ \\
\hline F12099 & $2 b$ & 2012 & Yangzhou & $R$ & I & Ser83Leu & Asp87Gly & His211Tyr & & Ser80lle & Ser129Pro & $a a c\left(6^{\prime}\right)-1 b-c r$ \\
\hline F12149 & $4 c$ & 2012 & Xuzhou & $R$ & S & Ser83Leu & & His211Tyr & & Ser80lle & Ser129Pro & $a a c\left(6^{\prime}\right)-1 b-c r$ \\
\hline F12150 & $2 a$ & 2012 & Xuzhou & $R$ & I & Ser83Leu & & His211Tyr & & Ser80lle & Ser129Pro & $a a c\left(6^{\prime}\right)-1 b-c r$ \\
\hline F13055 & $4 c$ & 2013 & Changzhou & $\mathrm{R}$ & $\mathrm{R}$ & Ser83Leu & & His211Tyr & & Ser80lle & Ser129Pro & anrs \\
\hline F13140 & $1 a$ & 2013 & Yangzhou & S & S & Ser83Leu & & His211Tyr & Gln517Arg & Ser80lle & & $a a c\left(6^{\prime}\right)-1 b-c r$ \\
\hline F14054 & $2 b$ & 2014 & Suqian & $\mathrm{R}$ & I & Ser83Leu & Asp87Asn & His211Tyr & & Ser80lle & & $a a c\left(6^{\prime}\right)-1 b-c r, a n r s$ \\
\hline F15099 & $2 a$ & 2015 & Taizhou & $\mathrm{R}$ & $R$ & Ser83Leu & Asp87Asn & His211Tyr & & Ser80lle & & gnrs \\
\hline F15100 & $2 a$ & 2015 & Taizhou & $\mathrm{R}$ & $\mathrm{R}$ & Ser83Leu & Asp87Asn & His211Tyr & & Ser80lle & & anrs \\
\hline F15101 & $2 a$ & 2015 & Taizhou & $R$ & $R$ & Ser83Leu & Asp87Asn & His211Tyr & & Ser80lle & & anrs \\
\hline F15105 & $2 a$ & 2015 & Taizhou & R & $R$ & Ser83Leu & Asp87Asn & His211Tyr & & Ser80lle & & anrs \\
\hline F15106 & $2 a$ & 2015 & Taizhou & $\mathrm{R}$ & $R$ & Ser83Leu & Asp87Asn & His211Tyr & & Ser80lle & Ser129Pro & anrs \\
\hline F15107 & $2 a$ & 2015 & Taizhou & $\mathrm{R}$ & $\mathrm{R}$ & Ser83Leu & Asp87Asn & His211Tyr & & Ser80lle & & anrs \\
\hline
\end{tabular}

NAL nalidixic acid, NOR norfloxacin; antibiotic susceptibility, $S$ susceptible, I intermediate resistant/susceptible, $R$ resistant

paper, we revealed a high rate of resistance to nalidixic acid (75.8\%), lower than that in 2006-2011 [7]. Additionally, we described here the worrisome fact that $37.0 \%$ of the S. flexneri isolates conferred resistance to norfloxacin. The present data showed a slight increasing of norfloxacin resistance rate, and, in contrast, a decreasing tendency of nalidixic acid resistance patterns, consistent with previous reports [7, 25].

Fluoroquinolone susceptibility profiles of $S$. flexneri varied between geographical locations. Nalidixic acid and norfloxacin resistance was documented in $100.0 \%$ and $22.3 \%$ of S. flexneri isolates in Anhui Province from 2005 to 2011 [6]. In Shanghai, all the S. flexneri showed nalidixic acid resistance [23]. In contrary, only $14.0 \%$ of S. flexneri was resistant to nalidixic acid and $8.4 \%$ to ciprofloxacin in Switzerland during 2004-2014 [26]. Overall, the results indicated the emerging problem that an increasing number of S. flexneri isolates have lost the natural susceptibility to fluoroquinolones. The worsening situation may be attributed to inappropriate prescription, easy access to antimicrobials and lack of local surveillance of antimicrobial resistance [25].

Besides, we observed the resistance patterns between serotypes. Serotypes $1 \mathrm{a}$ and $2 \mathrm{a}$ were demonstrated with higher rates of resistance to fluoroquinolones, which was consistent with that reported before [25]. Hence, antibiotic susceptibility testing performed at the start of an outbreak is highly recommended, and more prudent selection and use of fluoroquinolones are warranted.
Moreover, it is of great importance to conduct continuous and strong surveillance of fluoroquinolone resistance of Shigella in local areas for periodic updating of the local antibiograms.

This study revealed that substitutions within gyrA at Ser83 and His211 and Ser80 within parC were most frequently observed in the study years. Different with reported studies $[27,28]$, the presence of His211Tyr in gyrA, was of high prevalence among fluoroquinolone resistant $S$. flexneri isolates, and all the mutation was found with the concomitant presence of Ser83Leu mutation, the findings was similar to a study on $S$. flexneri serotype 4 s [29], indicating that His211Tyr have been found in several provinces around China. Previous study have shown that mutations in the primary target genes of fluoroquinolones in Shigella can lead to development of decreased susceptibility to fluoroquinolones [9], thus complicating the treatment of infections. Typically, resistance to nalidixic acid resulted mainly from a single amino acid substitution at either position 83 or position 87 of the gyrA gene [30]. Among the norfloxacin resistant isolates, $91.2 \%$ showed the presence of at least three substitutions in $g y r A$ and parC, and $81.8 \%$ presented the three common mutations Ser83Leu, Asp87Gly/Asn and Ser80Ile. Our results are consistent with the previous report that the presence of additional mutations in $g y r A$ at position 87 and parC mutation at either position 80 contributed to high-level fluoroquinolone resistance [9]. Unfortunately, the fluoroquinolone susceptible isolates 
also carried mutations within $\operatorname{gyr} A$ and $\operatorname{parC}$, leading to difficulty in clinical treatment.

Moreover, an interesting finding in our study was that the amino acid substitution at codon 87 in the parC gene varied significantly with serotypes and presented yearly variations. The mutation rate of Asp- 87 to Gly decreased annually during the four years, and Asp- 87 to Gly was highly prevalent in serotype $2 b$ and $1 \mathrm{a}$ while substitution of Asp-87 to Asn in serotype 2a was much more prevalent. However, limited reports on epidemiology of serotypes and mutations were published. Furthermore, less common, the substitution Gln91His and Ala81Pho were observed in $\operatorname{parC}$ and novel point mutation Gln517Arg in $g y r B$ gene was detected, which is worthy of notice.

There is scarce information on the prevalence of PMQR in Jiangsu Province while reports in other areas were common [27, 31-33]. The overall prevalence of PMQR in S. flexneri in the present study was $4.0 \%$, lower than that in Anhui and Henan, rates of $9.6 \%$ and $8.2 \%$, respectively [27, 31]. aac (6')-Ib-cr and qnrS were the most frequent $\mathrm{PMQR}$ determinants detected among $S$. flexneri. This agrees with previous reports that qnrS and $a a c\left(6^{\prime}\right)-I b-c r$ were the predominant PMQR determinants across two provinces located in Eastern China [31, 32]. Additionally, one of the $a a c\left(6^{\prime}\right)-I b-c r$ positive isolates co-harbored the qnrS gene. The frequency of $q n r B$ in this study was similar to the previous report in China $[28,31]$. Almost all of the PMQR-positive isolates carried uniform mutations Ser83Leu in gyrA and Ser80Ile in $\operatorname{parC}$, which may attribute to the promotion mutations within the QRDR, and result in resistance to fluoroquinolones. Furthermore, the qnrS-positive isolates in the present study demonstrated the report that the presence of gyrA mutation(s) and/or the qurS gene dramatically increased fluoroquinolone resistance for $S$. flexneri [34]. Given that Shigella harboring the qnrS gene are able to replicate efficiently in high concentrations of fluoroquinolones [34], the potential dissemination of fluoroquinolone resistance is evident. Seven of the PMQR-positive isolates were not norfloxacin resistant, which is in accordance with a previous published study in Switzerland [26], illustrating the potential for development of resistance in susceptible strains. Therefore, therapeutic efficiency of fluoroquinolones may be decreased [26, 33].

Our study had several limitations. First, Collection of S. flexneri may not a comprehensive of Shigella infection in Jiangsu Province because some people with shigellosis may not go to the hospital for medical care and diagnosis. Second, the $q n r$ alleles of $q n r B$ and $q n r S$ positive $S$. flexneri strains in this study are not detected, which make it difficult to better evaluate the prevalent trends of PMQR determinants in this area. Moreover, whether the His211Tyr in gyrA, Gln91His, Ala81Pho and Ser129Pro in parC and novel point mutation Gln517Arg in $\operatorname{gyr} B$ facilitate fluoroquinolone resistance is unknown and will be further studied in our next step.

\section{Conclusion}

Our study provides a baseline information on the prevalence in S. flexneri isolates of fluoroquinolone resistance and associated mechanisms including mutations in QRDR and PMQR determinants. Particularly, the significant finding of our study is the prevalence and distribution of PMQR genes in Shigella isolates in Jiangsu, China. Therefore, continuing active surveillance of antimicrobial resistance in Shigella is necessary and antimicrobial treatment must be updated regularly based on surveillance results.

\section{Abbreviations \\ CLSI: Clinical and Laboratory Standards Institute; NAL: Nalidixic acid; NOR: Norfloxacin; PMQR: Plasmid-mediated quinolone resistance; QRDR: Quinolone resistance-determining region}

\section{Acknowledgments}

We thank Professor Wen-chien Ko (Department of Internal Medicine, National Cheng Kung University Hospital, Tainan) for helpful comments on the work.

\section{Funding}

This work was funded by the Chinese National Natural Science Foundation (81471994), the Natural Science Foundation of Jiangsu Province (BK20151154), Jiangsu Provincial Medical Talent (ZDRCA2016053), Six talent peaks project of Jiangsu Province (WSN-135), and the Advanced Health Talents of Six-one Project of Jiangsu Province (LGY2016042).

\section{Availability of data and materials}

The datasets used and/or analysed during the current study are available from the corresponding author on reasonable request.

\section{Authors' contributions}

$\mathrm{TQ}$ and $\mathrm{HQ}$ were responsible for the study design. WF and PM collected microbiological data. LZ and CD analyzed the data. BG and XH contributed to the drafting of the manuscript. All authors have read and approved the final manuscript.

Ethics approval and consent to participate

Not applicable.

Consent for publication

Not applicable.

\section{Competing interests}

The authors declare that they have no competing interests.

\section{Publisher's Note}

Springer Nature remains neutral with regard to jurisdictional claims in published maps and institutional affiliations.

\section{Author details}

'Department of Laboratory Medicine, Affiliated Hospital of Xuzhou Medical University, Xuzhou 221002, China. ${ }^{2}$ Jiangsu Provincial Center for Disease Control and Prevention, Nanjing 210009, China. ${ }^{3}$ Medical Technology School, Xuzhou Medical University, Xuzhou 221004, China. 
Received: 1 April 2017 Accepted: 25 August 2017

\section{Published online: 19 September 2017}

\section{References}

1. Liu L, Johnson HL, Cousens S, Perin J, Scott S, Lawn JE, et al. Global, regional, and national causes of child mortality: an updated systematic analysis for 2010 with time trends since 2000. Lancet. 2012;379(9832):2151-61.

2. Wang XY, Tao F, Xiao D, Lee H, Deen J, Gong J, et al. Trend and disease burden of bacillary in China (1991-2000). Bull World Health Organ. 2006;84(7):561-8.

3. Walker CL, Rudan I, Liu L, Nair H, Theodoratou E, Bhutta ZA, et al. Global burden of childhood pneumonia and diarrhoea. Lancet. 2013:381(9875):1405-16.

4. Niyogi SK. Shigellosis. J Microbiol. 2005;43(2):133-43.

5. Livio S, Strockbine NA, Panchalingam S, Tennant SM, Barry EM, Marohn ME, et al. Shigella isolates from the global enteric multicenter study inform vaccine development. Clin Infect Dis. 2014;59(7):933-41.

6. Yang H, Chen G, Zhu Y, Liu Y, Cheng J, Hu L, et al. Surveillance of antimicrobial susceptibility patterns among Shigella species isolated in China during the 7-year period of 2005-2011. Ann Lab Med. 2013;33(2):111-5.

7. Qin T, Bi R, Fan W, Kang H, Ma P, Gu B. Novel mutations in quinolone resistance-determining regions of gyrA, gyrB, parC and parE in Shigella flexneri clinical isolates from eastern Chinese populations between 2001 and 2011. Eur J Clin Microbiol Infect Dis. 2016;35(12):2037-45.

8. Fàbrega A, Madurga S, Giralt E, Vila J. Mechanism of action of and resistance to quinolones. Microb Biotechnol. 2009:2(1):40-61.

9. Ruiz J. Mechanisms of resistance to quinolones: target alterations, decreased accumulation and DNA gyrase protection. J Antimicrob Chemother. 2003:51(5):1109-17.

10. Clinical and Laboratory Standards Institute. Methods for dilution and antimicrobial susceptibility tests for bacteria that grow aerobically, 9th ed. Approved standard M07-A9. Clinical and Laboratory Standards Institute, 2012. Wayne, PA.

11. Rahman M, Mauff G, Levy J, Couturier M, Pulverer G, Glasdorff N, et al. Detection of 4-quinolone resistance mutation in gyrA gene of Shigella dysenteriae type 1 by PCR. Antimicrob Agents Chemother. 1994:38(10):2488-91.

12. Chau TT, Campbell JI, Galindo CM, Van Minh HN, Diep TS, Nga TT, et al. Antimicrobial drug resistance of Salmonella enterica serovar typhi in asia and molecular mechanism of reduced susceptibility to the fluoroquinolones. Antimicrob Agents Chemother. 2007:51(12):4315-23.

13. Dutta S, Kawamura Y, Ezaki T, Nair GB, lida K, Yoshida S. Alteration in the GyrA subunit of DNA gyrase and the ParC subunit of topoisomerase IV in quinolone-resistant Shigella dysenteriae serotype 1 clinical isolates from Kolkata. India Antimicrob Agents Chemother. 2005;49(4):1660-1.

14. Robicsek A, Strahilevitz J, Sahm DF, Jacoby GA, Hooper DC. anr prevalence in ceftazidime-resistant Enterobacteriaceae isolates from the United States. Antimicrob Agents Chemother. 2006;50(8):2872-4

15. Wang M, Guo Q, Xu X, Wang X, Ye X, Wu S, et al. New plasmid-mediated quinolone resistance gene, $\mathrm{gnrC}$, found in a clinical isolate of Proteus mirabilis. Antimicrob Agents Chemother. 2009;53(5):1892-7.

16. Cavaco LM, Hasman H, Xia S, Aarestrup FM. anrD, a novel gene conferring transferable quinolone resistance in salmonella enterica serovar Kentucky and Bovismorbificans strains of human origin. Antimicrob Agents Chemother. 2009;53(2):603-8.

17. Pu XY, Pan JC, Wang HQ, Zhang W, Huang ZC, Gu YM. Characterization of fluoroquinolone-resistant Shigella flexneri in Hangzhou area of China. J Antimicrob Chemother. 2009;63(5):917-20.

18. Yamane K, Wachino J, Suzuki S, Arakawa Y. Plasmid-mediated gepA gene among Escherichia coli clinical isolates from Japan. Antimicrob Agents Chemother. 2008;52(4):1564-6.

19. Chang Z, Lu S, Chen L, Jin Q, Yang J. Causative species and serotypes of shigellosis in mainland China: systematic review and meta-analysis. PLOS One. 2012;7(12):e52515.

20. Mao Y, Cui E, Bao C, Liu Z, Chen S, Zhang J, et al. Changing trends and serotype distribution of Shigella species in Beijing from 1994 to 2010. Gut Pathog. 2013;5:21.

21. Ud-Din Al, Wahid SU, Latif HA, Shahnaij M, Akter M, Azmi IJ, et al. Changing trends in the prevalence of Shigella species: emergence of multi-drug resistant Shigella sonnei biotype $\mathrm{g}$ in Bangladesh. PLoS One. 2013;8(21):e82601.
22. von Seidlein L, Kim DR, Ali M, Lee H, Wang X, Thiem VD, et al. A multicentre study of Shigella diarrhoea in six Asian countries: disease burden, clinical manifestations, and microbiology. PLoS Med. 2006;3(9):e353.

23. Zhang J, Jin H, Hu J, Yuan Z, Shi W, Yang X, et al. Antimicrobial resistance of Shigella spp. from humans in shanghai, China, 2004-2011. Diagn Microbiol Infect Dis. 2014;78(3):282-6.

24. WHO. Antimicrobial Resistance: Global Report on Surveillance. Geneva. 2014 http://www.who.int/drugresistance/documents/surveillancereport/en/.

25. Xu Y, Zhuang L, Kang H, Ma P, Xu T, Pan S, et al. Prevalence, resistance patterns, and characterization of integrons of Shigella flexneri isolated from Jiangsu Province in China, 2001-2011. Eur J Clin Microbiol Infect Dis. 2016;35(8):1347-53.

26. Nüesch-Inderbinen M, Heini N, Zurfluh K, Althaus D, Hächler H, Stephan R. Shigella antimicrobial drug resistance mechanisms, 2004-2014. Emerg Infect Dis. 2016;22(6):1083-5.

27. Yang H, Duan G, Zhu J, Zhang W, Xi Y, Fan Q. Prevalence and characterisation of plasmid-mediated quinolone resistance and mutations in the gyrase and topoisomerase IV genes among Shigella isolates from Henan, China, between 2001 and 2008. Int J Antimicrob Agents. 2013;42(2):173-7.

28. Zhu YL, Yang HF, Liu YY, Hu LF, Cheng J, Ye Y, et al. Detection of plasmidmediated quinolone resistance determinants and the emergence of multidrug resistance in clinical isolates of Shigella in SiXian area. China Diagn Microbiol Infect Dis. 2013;75(3):327-9.

29. Yang C, Li P, Zhang X, Ma Q, Cui X, Li H, et al. Molecular characterization and analysis of high-level multidrug-resistance of Shigella flexneri serotype 4s strains from China. Sci Rep. 2016;6:29124.

30. Bhattacharya D, Bhattacharya H, Thamizhmani R, Sayi DS, Reesu R, Anwesh $M$, et al. Shigellosis in bay of Bengal Islands, India: clinical and seasonal patterns, surveillance of antibiotic susceptibility patterns, and molecular characterization of multidrug-resistant Shigella strains isolated during a 6-year period from 2006 to 2011. Eur J Clin Microbiol Infect Dis. 2014;33(2):157-70.

31. Liu Y, Hu L, Pan Y, Cheng J, Zhu Y, Ye Y, et al. Prevalence of plasmidmediated quinolone resistance determinants in association with betalactamases, 165 rRNA methylase genes and integrons amongst clinical isolates of Shigella flexneri. J Med Microbiol. 2012;61(Pt 8):1174-6.

32. Pu XY, Pan JC, Zhang W, Zheng W, Wang HQ, Gu YM. Quinolone resistancedetermining region mutations and the plasmid-mediated quinolone resistance gene anrS played important roles in decreased susceptibility to fluoroquinolones among Shigella isolates in southeast China between 1998 and 2013. Int J Antimicrob Agents. 2015;45(4):438-9.

33. Xiong Z, Li J, Li T, Shen J, Hu F, Wang M. Prevalence of plasmid-mediated quinolone-resistance determinants in Shigella flexneri isolates from Anhui Province, China. J Antibiot (Tokyo). 2010;63(4):187-9.

34. Thompson CN, Thieu NT, Vinh PV, Duc AN, Wolbers M, Vinh H, et al. Clinical implications of reduced susceptibility to fluoroquinolones in paediatric Shigella sonnei and Shigella flexneri infections. J Antimicrob Chemother. 2016;71(3):807-15.

\section{Submit your next manuscript to BioMed Central and we will help you at every step:}

- We accept pre-submission inquiries

- Our selector tool helps you to find the most relevant journal

- We provide round the clock customer support

- Convenient online submission

- Thorough peer review

- Inclusion in PubMed and all major indexing services

- Maximum visibility for your research

Submit your manuscript at www.biomedcentral.com/submit 